Revue d'histoire de l'Amérique française

Q4. REVUE D.HISTOIRE DE L'AMÉRIQUE FRANÇAISE

\title{
Un Canadien errant
}

\section{Louis-Marie Moreau, dit Coulon : hérésie et rébellion au Nouveau-Mexique}

\section{Sonya Lipsett-Rivera, Colin M. Coates et Pierre Desrosiers}

Volume 63, numéro 4, printemps 2010

URI : https://id.erudit.org/iderudit/1006006ar

DOI : https://doi.org/10.7202/1006006ar

Aller au sommaire du numéro

\section{Éditeur(s)}

Institut d'histoire de l'Amérique française

ISSN

0035-2357 (imprimé)

1492-1383 (numérique)

Découvrir la revue

Citer cet article

Lipsett-Rivera, S., Coates, C. M. \& Desrosiers, P. (2010). Un Canadien errant : Louis-Marie Moreau, dit Coulon : hérésie et rébellion au Nouveau-Mexique. Revue d'histoire de l'Amérique française, 63(4), 435-464.

https://doi.org/10.7202/1006006ar

\section{Résumé de l'article}

En 1739, un charpentier de Québec arrive au Nouveau-Mexique. Quatre ans plus tard, il est exécuté pour avoir suscité une rébellion indienne. Cet article examine les accusations portées contre cet homme, Louis-Marie Moreau dit Coulon, et les replace dans leurs contextes culturel et historique. Ayant critiqué les pratiques du catholicisme populaire dans la colonie espagnole, Moreau a soulevé la méfiance de plusieurs Indiens et Espagnols. En décrivant certaines pratiques guerrières de la région du Mississippi, il est de plus accusé d'avoir annoncé une invasion française. Même si les documents judiciaires transmettent mal ses propos, il est clair que Moreau eut de la difficulté à jauger les différences culturelles entre la Nouvelle-France et le Nouveau-Mexique. 


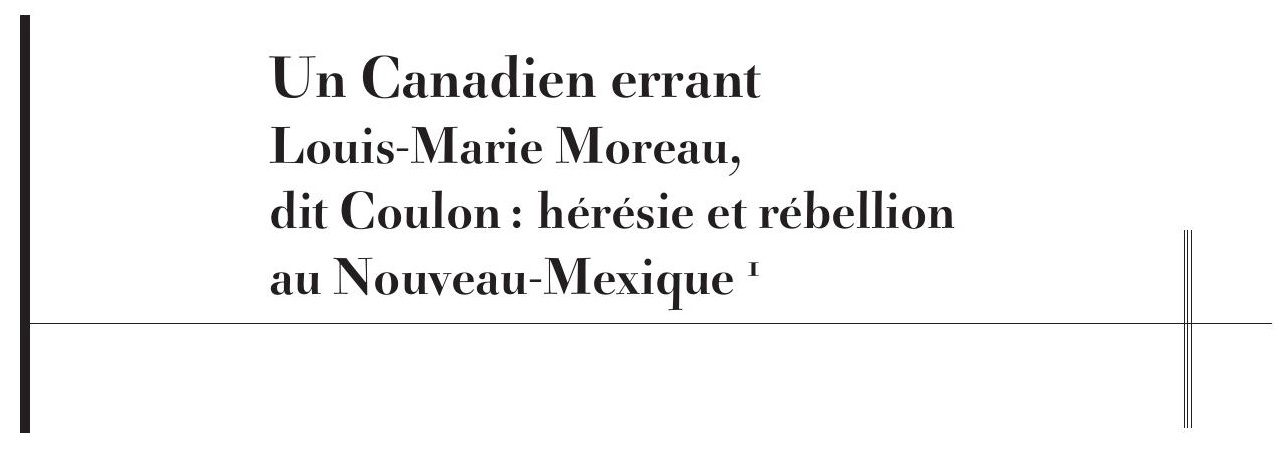

\author{
SONYA LIPSETT-RIVERA \\ Université Carleton \\ Colin Coates \\ Université York
}

RÉSUMÉ • En 1739, un charpentier de Québec arrive au Nouveau-Mexique. Quatre ans plus tard, il est exécuté pour avoir suscité une rébellion indienne. Cet article examine les accusations portées contre cet homme, Louis-Marie Moreau dit Coulon, et les replace dans leurs contextes culturel et historique. Ayant critiqué les pratiques du catholicisme populaire dans la colonie espagnole, Moreau a soulevé la méfiance de plusieurs Indiens et Espagnols. En décrivant certaines pratiques guerrières de la région du Mississippi, il est de plus accusé d'avoir annoncé une invasion française. Même si les documents judiciaires transmettent mal ses propos, il est clair que Moreau eut de la difficulté à jauger les différences culturelles entre la Nouvelle-France et le Nouveau-Mexique.

ABSTRACT • In 1739, a carpenter from Québec arrived in New Mexico. Four years later, he was executed for having fomented an Indian rebellion. This article examines the accusations made against this man, Louis-Marie Moreau dit Coulon, and places them in their cultural and historical contexts. Having criticized Catholic practices in the Spanish colony, Moreau attracted the distrust of various Indians and Spanish inhabitants. He described specific war

1. Nous souhaitons ici faire état de l'aide et des conseils que nous ont apportés de nombreux collègues lors de cette enquête sur les mystères entourant le voyage de Moreau : Charles Cutter, Thomas Wien, Jay Cassel, Susan Gray, Guillaume Teasdale, Brett Rushforth et les évaluateurs anonymes de cette publication. Nous remercions également M. John Osborne. Le Conseil de recherches en sciences humaines du Canada a assuré le financement de la recherche menée par Sonya Lipsett-Rivera. 
practices from the Mississippi region, and was also accused of having predicted a French invasion. Even if it is difficult to hear Moreau's voice in the judicial documents, he clearly failed to navigate the cultural differences between New France and New Mexico.

\begin{abstract}
$\grave{A}$
l'époque coloniale de l'Amérique du Nord, les Canadiens ont non seulement exploré les vastes territoires revendiqués par le roi de France mais ont poussé bien au-delà. Coureurs des bois indépendants, militaires ou «engagés» par les commerçants de fourrures, ils se sont aventurés dans de nombreux coins du continent. Ce faisant, ils ont fait contact avec de nombreux peuples aborigènes, de même qu'avec d'autres Européens, et se sont efforcés, avec plus ou moins de succès, d'éviter les méprises potentiellement catastrophiques nées du choc des cultures. L'historiographie actuelle s'est jusqu'ici penchée, pour l'essentiel, sur les explorations réussies, les Canadiens agissant alors comme intermédiaires culturels et parvenant souvent à dégager une synthèse des coutumes et des croyances françaises et autochtones ${ }^{2}$. Mais si de nombreux voyageurs nouèrent ainsi des liens heureux avec les peuples autochtones, tous ne furent pas aussi chanceux.
\end{abstract}

Cet article porte sur le cas de Louis-Marie Moreau, dit Coulon (ou Coulonge), menuisier de la ville de Québec arrivé au Nouveau-Mexique en 1739. Son aventure regorge de complications, car il échoua à composer correctement avec les diverses collectivités rencontrées sur sa route. Les raisons de cet échec sont particulièrement intéressantes dans la mesure où elles révèlent à la fois la nature conservatrice de son bagage culturel et ses tentatives d'innover.

Appuyés sur des documents qui avaient été mal classés dans les archives de Mexico, nous examinerons donc le cas d'un Canadien qui finit ses jours loin des limites territoriales de l'Amérique du Nord française. Entre 1739 et 1743, Moreau s'installa au Nouveau-Mexique, région frontalière du nord de la Nouvelle-Espagne. Ses critiques des pratiques locales de la foi catholique, de même que l'emploi de quelques tours de magie et de vagues menaces d'une attaque française, suscitèrent des soupçons chez

2. Richard White, The Middle Ground: Indians, Empires, and Republics in the Great Lakes Region, 1650-1815 (New York: Cambridge University Press, 1991), Gilles Havard, Empire et métissages: Indiens et Français dans le Pays d'en Haut, 1660-1715 (Sillery: Septentrion, 2003); Gilles Havard et Cécile Vidal, Histoire de l'Amérique française (Paris: Flammarion, 2006), 347-384 ; Carolyn Podruchny, Voyageurs et traiteurs de fourrures en Amérique du Nord (Québec: Les Presses de l'Université Laval, 2009). 
ses nouvelles relations. Alors même qu'il s'efforçait d'expliquer ce qu'il comprenait des rituels autochtones, il refusa d'admettre que le catholicisme puisse, sous sa forme populaire, comporter diverses expressions. Accusé d'hérésie et de rébellion, il fut fusillé sur la place publique de Santa Fe.

\section{L'EXPÉDITION MALLET}

En 1739, un groupe de négociants en fourrures, dirigé par Pierre et Paul Mallet, atteignit le Nouveau-Mexique depuis la région de l'Illinois. Ils étaient probablement les premiers Européens à emprunter la Piste de Santa Fe, qui allait finalement devenir la principale voie d'accès menant de la vallée du Mississippi au Nouveau-Mexique ${ }^{3}$. Ils avaient été précédés dans le sud-ouest, depuis un demi-siècle, par divers Français et Canadiens attirés par des rumeurs de mines d'argent et des rêves de commerce ou habités du désir d'étendre les influences françaises à l'intérieur du continent. De telles entreprises furent souvent menées à titre privé, dont notamment celles des déserteurs de l'équipe de René Robert Cavelier de La Salle lors des explorations de 1693 (après la mutinerie de 1687 au cours de laquelle La Salle fut assassiné), de Louis Juchereau qui mena une expédition commerciale en 1713-1714 et d'Étienne de Bourgmont qui tint, en 1724, des pourparlers avec les Padoucas (probablement des Apaches) aux frontières du Nouveau-Mexique ${ }^{4}$. Aucune expédition ne pouvait être menée à terme sans la coopération des peuples autochtones et les Français croyaient pouvoir largement compter sur l'amitié de divers peuples. De Bourgmont rapporta, au terme de son expédition, les paroles de ses hôtes apaches: "quand les François viendront nous voir, nous les recevrons bien, et en cas qu'ils veuillent aller chez les Espagnols pour y traiter, nous les y conduirons. Il n'y a que douze jours de marche de nostre village ${ }^{5}$.»

À la différence de l'expédition de Bourgmont, celle de Mallet fut entreprise sans autorisation officielle, bien que les autorités françaises de la Louisiane se soient, par la suite, montrées fort intéressées à ses décou-

3. Dans Along Ancient Trails: The Mallet Expedition of 1739 (Niwot, Colorado: University Press of Colorado, 1995), Donald J. Blakeslee en retrace méticuleusement le parcours.

4. Noel M. Loomis et Abraham P. Nasatir, Pedro Vial and the Roads to Santa Fe (Norman: University of Oklahoma Press, 1967), chap. 3 ; Henri Folmer, «De Bourgmont's Expedition to the Padoucas in 1724, the First French Approach to Colorado", The Colorado Magazine, 14, 4 (juillet 1937): 121-128.

5. «Demandes du Sieur de Bourgmont pour aller faire la paix avec les nations voisines du Nouveau-Mexique", dans Pierre Margry, dir., Découvertes et Établissements des Français dans l'Ouest et dans le Sud de l'Amérique Septentrionale (1614-1754) (Paris: Maison Neuve et Ch. Leclerc, 1888), $6^{\mathrm{e}}$ partie, 436. 
vertes. Accompagnés de six ou sept «Canadiens ${ }^{6}$ », les frères Mallet adoptèrent, conseillés par des Autochtones demeurés anonymes, un itinéraire différent de celui de leurs prédécesseurs. Ils quittèrent le pays des Illinois le 29 mai 1739, empruntant diverses rivières qui les menèrent dans des territoires arides et dénués d'arbres. Le 24 juin, ils aperçurent des rochers portant des inscriptions en espagnol, premiers signes d'une occupation du territoire par l'Espagne. Un esclave Lalitan (Comanche) évadé leur fit franchir une partie de la distance vers le Nouveau-Mexique. Le passage d'une rivière à gué leur coûta neuf chevaux et toute la marchandise qu'ils transportaient à des fins de commerce. Finalement, le 15 juin, ils prirent contact avec les Espagnols. Bien reçus par leurs hôtes, ils tentèrent d'établir des liens avec le lointain avant-poste de Nouvelle-Espagne. Ils déclarèrent au gouverneur don Gaspar Domingo de Mendoza qu' « ils venoient à dessein d'introduire un commerce avec les Espagnols de ce royaume pour l'estroite union qu'il y a entre les deux couronnes de France et d'Espagne». En accomplissant ce voyage, ils avaient pour but de "descouvrir ce royaume et luy donner communication avec les colonies de la NouvelleOrléans et Canada ${ }^{7}$.

Leur arrivée au Nouveau-Mexique provoqua une forte surprise et guère moins de méfiance. Un des prêtres qui assista à l'événement affirma, des années plus tard, que les ayant vus «sin insignia, ni señal de christianos, nunca hisso juicio de que fueron Catholicos" (sans insigne ou autre signe montrant qu'ils étaient chrétiens, il n'était pas d'avis qu'ils étaient catholiques) ${ }^{8}$. Pourtant, les frères Mallet se comportèrent très correctement, ce dont fit état le gouverneur: "[ils] ont esté chez moy et à ma table, [et] ont tenu une conduite très régulière et très chrestienne ${ }^{9}$ ». Quelque dix ans plus tard, Pierre Mallet justifia son expédition en élaborant sur ses prétentions antérieures: "dans l'année ' 40 [sic], de même que huit autres compagnons de la ville de Canada, désireux de voir le pays et de chercher emploi ou occupation pour nos familles, et encouragé par des rapports faisant état de la proximité des Espagnols, sommes venus en passant par

6. Les documents de l'époque mentionnent un groupe de huit personnes et omettent souvent le neuvième, nommé Jean-Baptiste Alarie. D. Blakeslee, op. cit., 24.

7. «Copie d'un certificat donné à Santa-Fé aux sept François par le général Jean Paëz Hurtado [sic], alcalde, major et capitaine de guerre de cette ville capitale de Santa-Fé et sa jurisdiction, lieutenant de gouverneur et capitaine général de ce royaume du Nouveau-Mexique et ses Provinces », 30 avril 1740, dans P. Margry, op. cit., 463.

8. AGN, Criminal, vol. 577, exp. 10, fol. 385.

9. "Copie d'un certificat», dans P. Margry, op. cit., 464. 
Isla Negro, ou un village indien en Nouvelle France, à ce royaume du Nouveau Mexique ${ }^{10}$ ».

Ne sachant quel comportement adopter envers ces visiteurs, les autorités locales demandèrent conseil, par lettre, au vice-roi qui résidait à Mexico. La réponse leur parvint neuf mois plus tard. Le vice-roi ne voulait pas que les Canadiens retournent dans les territoires sous domination française, mais les encourageait plutôt à demeurer au Nouveau-Mexique. S'ensuivit, chez les Canadiens, la crainte d'être forcés de s'enfoncer plus avant dans l'ouest, où ils rencontreraient «des hommes vestus de soye, qui habitent de grandes villes sur le bord de la $\operatorname{mer}^{11}$ ».

En dépit des instructions du vice-roi, les Canadiens furent autorisés à quitter le Nouveau-Mexique le $1^{\text {er }}$ mai 1740 . Le 13, trois d'entre eux se séparèrent du groupe afin de retourner dans le territoire des Illinois. Quatre autres, dont les frères Mallet, prirent la direction de la NouvelleOrléans. En route, ils rencontrèrent un groupe de cinq Padokas qui s'enfuirent de frayeur. Le 19 juin, ils abandonnèrent les 18 chevaux qu'il leur restait et construisirent des canots d'écorce à bord desquels ils descendirent la rivière. La rivière dite "Canadian River", qui coule du Colorado actuel jusqu'à l'Oklahoma en passant par le Texas, tient probablement son nom de ce voyage des frères Mallet. Cinq jours plus tard, ils rencontrèrent un groupe de chasseurs canadiens à la jonction de la rivière Arkansas. Après avoir passé quelques jours à chasser en leur compagnie, ils poursuivirent leur voyage jusqu'au fort Arkansas, puis vers la NouvelleOrléans où ils n'arrivèrent qu'en mars $1741^{12}$.

Entre-temps, deux membres de l'expédition avaient choisi de demeurer au Nouveau Mexique: Jean-Baptiste Alarie, né à Montréal, devint ainsi, dans les dossiers espagnols, Juan Bautista Alari ou Alarid. Il pratiqua le métier de barbier et engendra une famille distinguée et très en vue. En 1748, le gouverneur don Joaquin Codallos y Rabal, confirma que Alarie, "homme prospère, [s'était] comporté honorablement ${ }^{13}$ ".

10. Charles Wilson Hackett, dir. et trad., Pichardo's Treatise on the Limits of Louisiana and Texas (Freeport, NY : Books for Libraries Press, 1971 [1941]), 334. Il est possible que "Isla Negro » soit une traduction en espagnol du mot français «Illinois», dès lors devenu "Île Noire».

11. «Extrait du Journal de ce voyage, présenté à MM. de Bienville, Gouverneur, et Salmon, Intendant de la Louisiance», dans P. Margry, op. cit., 459.

12. "Jean-Baptiste LeMoyne de Bienville et Edmé-Gatien Salmon au ministre de la Marine», 30 avril 1741 , ibid., 466.

13. "Don Joaquin Codallos y Rabal au "gouvernement supérieur de la Nouvelle Espagne , 4 mars 1748, dans Ralph Emerson Twitchell, dir., The Spanish Archives of New Mexico (Cedar Rapids, Iowa: Torch Press, 1914), vol. 1, 151. 
Le second Canadien s'appelait Louis-Marie Moreau dit Coulon. Selon les documents produits pour l'Inquisition, il épousa María de Guadalupe, fille d'une prostituée («mujerilla pública»), bien que la jeune femme porte le nom de Juana Muñoz dans le registre paroissial où est consignée la date de leur mariage $(12 \text { octobre } 1740)^{14}$. María de Guadalupe était déjà mariée à Juan Francisco Muñoz, lequel quitta Santa Fe en 1740 pour accompagner quelques religieux à Mexico. Comme elle était enceinte au départ de Muñoz, celui-ci se sentit bientôt coupable d'abandonner son épouse; parvenu à Chihuahua, il sollicita du chef de groupe la permission de revenir au Nouveau-Mexique, sa femme devant bientôt donner naissance. Or María de Guadalupe prétendit que le père de l'enfant n'était pas Muñoz mais un muletier nommé Gómez, de sorte que Muñoz revenait déshonoré ${ }^{15}$. Il est clair que Moreau avait frayé avec les éléments marginaux de la colonie. Mentionnant le nom de María de Guadalupe, le gouverneur Domingo de Mendoza ajouta «buen nomer pero malos echos» (un bon nom pour de mauvaises actions), allusion probable au fait qu'elle portait le prénom de la Vierge Marie ${ }^{16}$. Leonor Montaño, qui témoignera plus tard des activités de Moreau, était l'épouse d'un charretier; la parenté des métiers suggère peut-être l'existence d'un lien entre Montaño et l'épouse de Moreau.

Nous n'en savons guère sur les activités de Moreau au cours des deux années suivantes. En furent au courant les gens d'au moins cinq villages (Isleta, Tesuque, Picuris, Laguna et Zuni), ainsi que ceux des deux villes coloniales d'Albuquerque et de Santa Fe. Sa connaissance de la langue espagnole demeure un mystère, mais il est peu probable qu'il ait, en l'espace de quatre années, maîtrisé les quatre langues Pueblo distinctes parlées à travers l'immense territoire où il était connu. Selon le gouverneur, Moreau entreprit de fomenter une rébellion en octobre 1742: "Cette personne, arrivée ici, a tenté par de subtiles intrigues d'inciter à la révolte les Indiens de ce royaume ${ }^{17}$. Sachant la fragilité du pouvoir espagnol sur les villages, les activités de Moreau étaient, en effet, menaçantes. Mis au fait de cette agitation naissante en mai 1743, après qu'un Indien nommé

14. Fray Angélico Chávez, Origins of New Mexico Families in the Spanish Colonial Period (Santa Fe: The Historical Society of New Mexico, 1954), 239.

15. AGN, Criminal, vol. 577, exp. 10, folio 375v; fol. 359-359v.

16. Ibid., fol. 360 .

17. Charles Wilson Hackett, dir., Historical Documents relating to New Mexico, Nueva Vizcaya, and Approaches Thereto, to 1773 (Washington, DC: Carnegie Institution of Washington, 1937), vol. 3, 391. 
Francisco El Coyote ${ }^{18}$ eut fait état de plusieurs accusations, le gouverneur ordonna d'appréhender Moreau à Tesuque et de l'amener à Santa Fe. Le gouverneur don Gaspar Domingo de Mendoza le condamna à mort; le 29 mai, Moreau fut amené à l'église pour s'y confesser et se préparer à mourir. L'exécution devait avoir lieu le 31, par arrachement du cour à travers le dos («sacado por los espaldas»). Cependant un prêtre du lieu, nommé Pedro del Pino, avait demandé au gouverneur de remettre l'exécution à quelques jours. Le gouverneur opposa d'abord un refus, invoquant le fait que la date de l'événement avait été fixée. En fait, la raison tenait plus probablement à ce que les autorités avaient ordonné aux indios principales de toutes les communautés environnantes de venir à Santa Fe assister à l'exécution. Conformément à ce qu'était la culture juridique punitive du début de l'ère moderne, les autorités allaient rendre là une justice exemplaire qui servirait de leçon à quiconque serait tenté de manifester un semblable esprit de rébellion.

L’exécution fut retardée. À cause des allégations portées contre Moreau, on invoqua la compétence de l'Inquisition à examiner les supposées hérésies de l'accusé. On entreprit donc de recueillir chez divers individus, espagnols et autochtones, des témoignages prouvant qu'il avait pratiqué la magie, critiqué les pratiques religieuses et menacé la souveraineté espagnole sur le territoire. Moreau étant déjà condamné à mort, rien ne porte à croire que ces témoignages avaient pour unique but de le convaincre faussement d'activités illicites. Les témoins apportèrent plutôt des preuves relatives à ses positions religieuses. La multiplicité des métiers et des origines des témoins laisse à penser qu'il ne s'agissait pas là de monter un dossier ordonné et systématique contre Moreau. En outre, il est peu probable qu'il ait été impliqué dans un trafic d'armes avec les peuples autochtones, ce qui eût été considéré menaçant par les autorités coloniales, car l'expédition Mallet avait perdu toute sa marchandise avant même d'atteindre la colonie. Aucun trafic d'armes n'est mentionné dans les documents d'archives concernant Moreau.

On jugea finalement que l'Inquisition n'avait aucun rôle à tenir dans l'affaire et le menuisier de Québec fut fusillé sur la place publique de Santa Fe le 19 octobre 1743. Après quoi les documents afférents parvinrent à la Archivo General de la Nación, à Mexico, et furent insérés dans un dossier

18. Selon Ross Frank, le nom de "coyote» était accolé aux enfants nés des esclaves indiennes. Ross Frank, "Demographic, Social, and Economic Change in New Mexico» dans Robert H. Jackson, dir., New Views of Borderlands History (Albuquerque: University of New Mexico Press, 1998), 56-57. 
d'une tout autre nature (portant sur une poursuite concernant des droits sur les ressources hydrauliques au centre du Mexique). Sur les accusations et sur l'exécution de Moreau, les historiens n'avaient ainsi pour tout partage que quelques renseignements sommaires ${ }^{19}$.

Parce qu'ils apportent une version plus détaillée des allégations relatives aux activités et aux croyances de Moreau, les documents retrouvés aux archives nous permettent de mieux saisir ses tentatives de s'intégrer à son nouveau pays. Ses activités, cependant, demeurent obscures. À supposer qu'il ait exécuté les quelques tours de magie dont on l'accusait, il laisse l'impression d'un individu menaçant mais incohérent, parvenant mal à expliquer la signification de ses actes. Néanmoins, folie et communication fautive n'excluent pas forcément une certaine logique, ainsi que l'a montré Eric Van Young à propos de la chimérique rébellion du Messie Fou de Durango dans les premières années du XIX ${ }^{\mathrm{e}}$ siècle mexicain ${ }^{20}$. Après avoir fourni un aperçu des origines de Moreau en Nouvelle-France, nous nous pencherons sur le contexte de ses activités et sur les interprétations qu'en donna le gens du Nouveau-Mexique.

\section{LES ORIGINES}

Quelques rares documents paroissiaux et actes judiciaires et notariés résument la vie à Québec de Louis-Marie Moreau, dit Coulon, et précisent ses antécédents familiaux et son emploi. Il naquit le 28 novembre 1705 , quatrième enfant, et deuxième fils, de Louis Moreau et de Catherine Bonhomme. Selon le registre paroissial, trois autres enfants lui succédèrent, deux frères et une sœur. Dans le recensement de 1716, le père est décrit comme «fermier» et la famille compte aussi un Jean Moreau, âgé de 50 ans, sûrement apparenté au père et décrit comme «domestique ${ }^{21}$.

19. Ainsi, Hubert Bancroft, History of Arizona and New Mexico, 1530-1888 (Albuquerque : Horn and Wallace, 1962), 233, raconte qu'un membre de l'expédition Mallet, nommé Jean d'Alay (Alarie), se maria et devint un bon citoyen, mais que Louis-Marie Moreau se maria, s'attira des ennuis et fut fusillé sur la place publique. D’autres brèves allusions se trouvent, entre autres, chez David J. Weber, The Spanish Frontier in North America (New Haven: Yale University Press, 1992), 196 et A. P. Nasatir, Before Lewis and Clark: Documents Illustrating the History of the Missouri, 17851804, vol. 1 (St. Louis: St. Louis Historical and Document Foundation, 1952), 27-28.

20. Eric Van Young, "Millennium on the Northern Marches: The Mad Messiah of Durango and Popular Rebellion in Mexico, 1800-1815 ", Comparative Studies in Society and History, 28 (1986): 385-413.

21. Hubert Charbonneau et Jacques Légaré, dir., Répertoire des actes de baptême, mariage, sépulture et des recensements du Québec ancien, vol. 8. 
Il semble que Louis-Marie ait suivi les traces de son frère aîné en apprenant le métier de menuisier et, plus tard, en menant des incursions à l'intérieur du continent. À l'âge de 23 ans, Joseph-Louis, mentionné sous le nom de Louis, signa en 1721 un engagement à se rendre à Détroit. En compagnie de Pierre Juneaux, de Québec, de François Couder, de Contrecour et de Jean-Baptiste Langtôt [Lanctôt] de Boucherville, il s'engagea par contrat auprès de Alphonse de Tonty, capitaine dans les Troupes de la Marine. Contre un ballot de peaux de castor d'une valeur de 60 livres, les quatre hommes promettaient de se rendre par canot à Détroit. Louis signa le contrat de son propre nom ${ }^{22}$. De retour à Québec, il sera plus tard décrit comme "charpentier» dans le recensement de 1744 .

Louis-Marie Moreau est nommément mentionné dans un document de 1731. Cette année-là, une ordonnance condamna la veuve Dumontier à payer 39 livres pour l'achat de "madriers» obtenus de Coulange Moreau, menuisier de Québec ${ }^{23}$. Bien qu'il soit difficile de retracer son parcours, tout porte à croire que Louis-Marie avait alors terminé sa période d'apprentissage - sous le Régime français, l'âge moyen des apprenti-charpentiers était légèrement supérieur à $18 \mathrm{ans}^{24}$. Un document notarié, rédigé alors qu'il avait atteint l'âge de 26 ans, le qualifie de "maitre menuisier». En mai 1732, il paraphe avec René Letarte, constructeur de maisons de Neuville, une entente par laquelle il fournira un meuble de pin. À la différence de son frère, Louis-Marie ne savait pas signer de son nom ${ }^{25}$.

Tout au long de son histoire, la Nouvelle-France fut en manque d'artisans qualifiés ${ }^{26}$, bien que les menuisiers aient fourni le plus fort contingent de cette main-d'œuvre. Vivant habituellement chez leur maître durant leurs années d'apprentissage, les artisans étaient mieux traités que ne l'étaient leurs équivalents en France. Il arrivait souvent, dans la colonie, qu'on leur accorde un salaire ${ }^{27}$. Bien que la main-d'œuvre qualifiée ait été

22. Bibliothèque et Archives nationales du Québec à Québec [BANQ-Q], Greffe Jacques David, Mf 2117, 6 août 1721 .

23. Bibliothèque et Archives Canada [BAC], MG8 A6, vol. 11, p. 139-140, 18 déc. 1731, bobine C-13509.

24. Jean-Pierre Hardy et David-Thierry Ruddel, Les Apprentis-artisans à Québec, 1660-1815 (Montréal: Presses de l'Université du Québec, 1977), 26.

25. BANQ-Q, Greffe Panneton, 14 mai 1732.

26. La même pénurie affligeait le Nouveau-Mexique. En 1748, le gouverneur sollicita du vice-roi la permission d'autoriser trois déserteurs français (respectivement charpentier, tailleur et barbier) à demeurer à Santa Fe, où l'on manquait désespérément d'hommes de métier. Herbert E. Bolton, «French Intrusions into New Mexico, 1749-1752», dans John Francis Bannon, dir., Bolton and the Spanish Borderlands (Norman: University of Oklahoma Press, 1964), 157-158.

27. Sur les apprentis au Québec, J.-P. Hardy et D. T. Ruddel, op. cit., 22-51. 
rare, et donc très prisée, l'appel de la traite des fourrures n'en diminuait pas pour autant; les profits réalisés sur le court terme dépassaient, tout simplement, les revenus d'un ouvrier, fût-il qualifié28.

De 1732 à 1739, le nom de Louis-Marie Moreau disparaît des archives. En 1735, le gouverneur et l'intendant entérinent un acte du 11 juillet 1734, par lequel est concédé une terre de quatre arpents, sise à Détroit, "au nommé Moreau "; il s'agit possiblement de Louis-Marie ou de l'un de ses frères $^{29}$. À cette époque, Détroit était devenu un poste de traite auquel s'était greffée une petite population d'agriculteurs français. Dans cette agglomération, Moreau eût été au nombre des rares habitants originaires de Québec. Au XVIII ${ }^{e}$ siècle, la plupart des migrants venaient de Montréal et leur occupation directement liée à la traite des fourrures ${ }^{30}$. Outre Détroit, d'autres communautés françaises prenaient racine à l'intérieur du continent, formant ainsi un réseau peu structuré entre la vallée du Saint-Laurent et la colonie de la Louisiane.

Il est aussi possible que Moreau se soit trouvé, en conséquence de la guerre contre les Chicasas, en territoire des Illinois à la fin des années 1730 . En 1736 et de nouveau en 1739, le gouverneur Charles de Beauharnois de la Boische envoya un contingent mixte de soldats français, canadiens, amérindiens et africains se joindre aux forces de Jean-Baptiste LeMoyne de Bienville, gouverneur de la Louisiane, qui combattait les Chicasas ${ }^{31}$. Les autorités françaises entretenaient depuis longtemps des relations hostiles avec ce peuple autochtone. Selon un observateur jésuite, les Chicasas étaient une "Nation brave, mais perfide, et peu connue des Fran-

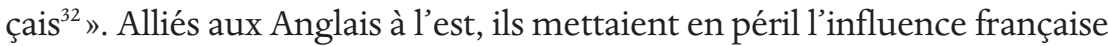
sur le Mississippi et, en particulier, les voies d'approvisionnement qui menaient de la région des Illinois jusqu’en Louisiane. Miliciens canadiens,

28. Peter Moogk, The Craftsmen of New France (Université de Toronto: thèse de doctorat, 1973), 231

29. P.-G. Roy, dir., Inventaire des insinuations du Conseil Souverain de la Nouvelle-France (Beauceville: L’Éclaireur, 1921), 205-206.

30. Lina Gouger, «Migrer à Détroit au XVIIe siècle: La part du monde rural dans le peuplement d'une zone frontière", dans Gérard Bouchard, John A. Dickinson et Joseph Goy, dir., Les Exclus de la Terre en France et au Québec, XVII ${ }^{e}$ XX ${ }^{e}$ siècles (Sillery: Septentrion, 1998), 161-174.

31. Patricia Dillon Woods, French-Indian Relations on the Southern Frontier, 1690-1763 (Ann Arbor: UMI Research Press, 1980); Dale Miquelon, New France, 1701-1744: "A Supplement to Europe" (Toronto: McClelland and Stewart, 1987), 188; Louise Dechêne, Le Peuple, l'État et la Guerre au Canada sous le Régime français (Montréal: Boréal, 2008), 484-487.

32. Reuben Gold Thwaites, dir., The Jesuit Relations and Allied Documents (Cleveland: Burrows, 1900), vol. 68, 200. 
soldats français, guerriers autochtones et esclaves africains combattirent ensemble pour vaincre les Chicasas, mais sans y parvenir.

Peut-être Moreau a déserté la Milice alors qu’il vivait dans la région, ce qui expliquerait son désir de demeurer au Nouveau-Mexique. Il est certain que le gouverneur Bienville n'était guère satisfait de l'attitude des miliciens canadiens, qu'il qualifia de "naturellement un peu mutins ${ }^{33}$ ». Dans une allusion aux déserteurs français arrivés au Nouveau-Mexique après l'expédition Mallet, Felipe de Sandoval, fonctionnaire du gouvernement, expliqua l'attrait qu'exerçait ce lieu lointain: «La confiance et l'excellent accueil dont profitent les Français chez les Comanches, grâce aux recommandations des Jumanos, de même que les renseignements qu'ils ont obtenus, des mêmes peuples et d'autres Français, concernant nos établissements [...] sont la cause de la désertion et de la fuite de ces Français vers ces possessions ${ }^{34}$.» Que Moreau ait fréquenté la région des Illinois comme déserteur de la Milice ou comme commerçant de fourrures, il est clair, selon les documents judiciaires du Nouveau-Mexique, qu'il jouissait de très nombreux contacts chez les peuples autochtones et qu'il s'efforça d'employer, dans ses efforts pour communiquer avec les Amérindiens du Nouveau-Mexique, des pratiques apprises dans la région des Illinois ou du Mississippi. Leur emploi dans un autre contexte culturel échoua lamentablement.

Quoiqu'il en soit, Louis-Marie avait atteint la région des Illinois en 1739, au Fort des Chartres, point de départ probable de l'expédition Mallet ${ }^{35}$. Plusieurs petits villages amérindiens et français s'étaient créés, dans la région, autour du réseau hydrographique du Mississippi, loin de l'autorité immédiate du gouvernement français. Disposant d'une forte proportion d'esclaves, ces villages à dominance française se consacraient à la culture de céréales destinées aux établissements situés en aval du Mississippi. Des villages français avaient ainsi surgi en bordure de campements autochtones et un réseau complexe de relations s'était développé entre les uns et les autres ${ }^{36}$.

Pour leur famille demeurée à Québec, Louis-Marie et ses frères avaient quitté la colonie et rien ne portait à croire qu'ils y reviennent un jour. Leur

33. Cité dans C. E. O’Neill, «Le Moyne de Bienville, Jean-Baptiste», Dictionnaire biographique du Canada, vol. 3 (Québec, Les Presses de l’Université Laval).

34. Felipe de Sandoval, cité dans C. W. Hackett, dir. et trad., Pichardo's Treatise..., op. cit., 326.

35. D. Blakeslee, op. cit., 56.

36. Winstanley Briggs, «Le Pays des Illinois», William \& Mary Quarterly, $3^{\mathrm{e}}$ série, 47, 1 (janvier 1990) : 30-56; Carl J. Ekberg, French Roots in the Illinois Country: The Mississippi Frontier in Colonial Times (Urbana: University of Illinois Press, 1998). 
père mourut le 29 octobre 1735 et Catherine Bonhomme, leur mère, le 15 juillet 1747. Lors de ces décès, trois de leurs fils étaient absents, disséminés quelque part sur le continent. Le plus âgé, Louis Moreau, dressa la liste des avoirs de ses parents. On y relève des terres à Sainte-Foy ainsi qu'une maison et un lot dans la haute ville de Québec. Soustraction faite des années d'arrérages dus à la famille Dartigny pour les terrains de Sainte-Foy et de Québec et après avoir divisé l’héritage par sept, chacun des frères absents allait recevoir la somme de 537 livres et 6 sols $^{37}$. Le document de 1748, qui enregistrait le droit de Louis-Marie à l'héritage, nota, sans plus, que Louis-Marie et ses frères, Eustache et Valentin, étaient "absents de cette colonie ${ }^{38}$ ». Eustache, inscrit comme maître maçon à Kaskaskia en 1739, et Valentin, prirent tous deux épouse et s'installèrent en Louisiane ${ }^{39}$. Si la mort de Louis-Marie au Nouveau-Mexique fut probablement plus dramatique, il se peut que sa famille de Québec ait également ignoré ce qu'il advint de ses deux jeunes frères.

\section{LA CRAINTE DES FRANÇAIS AU NOUVEAU-MEXIQUE}

Au début du XVIII ${ }^{\mathrm{e}}$ siècle, le Nouveau-Mexique formait la lisière nord de l'établissement espagnol dans le Nouveau Monde; sur ce territoire, les Espagnols avaient tenté - avec un succès tout relatif - d'imposer leur autorité chez les Indiens Pueblo. En 1680, ces derniers s'étaient révoltés et avaient momentanément chassé les conquérants. Revenus pour de bon, les Espagnols ne parvinrent pas à s'imposer totalement. Malgré ces difficultés à régenter la population autochtone, ils saisissaient parfaitement l'importance stratégique du territoire, zone tampon entre eux et d'autres puissances coloniales.

De leur côté, les Français avaient perçu, eux aussi, les occasions d'enrichissement qu'offrait le Nouveau-Mexique; certaines discussions offi-

37. BANQ-Q, Greffe Jacques Pinguet, inventaire après décès, 26 juillet 1747; BANQ-Q, Greffe DuLaurent, 4 octobre 1748.

38. BANQ-Q, Greffe Dulaurent, 12 mai 1748. De même, lorsque la sœur de Jean-Baptiste Alarie vendit une propriété au nom de ses frères, les documents d'enregistrement mentionnent Jean-Baptiste et François comme «leurs frères absent, de présent aux Ilinois». Ils avaient antérieurement fait «donation » à leur sœur de leur prétention à l'héritage. BANQ-Montréal, Greffe Danré de Blanzy, 30 juin 1740; ANQ-M, Insinuations de la Prévôté, 6 juin 1740.

39. Natalia Maree Belting, Kaskaskia under the French regime (Urbana: University of Illinois Press, 1948), p. 62, note 78. Les recherches généalogiques portent à croire que les deux hommes prirent épouse dans les territoires de l'intérieur. Valentin épousa Marie-Jeanne Lafleur et décéda en 1782 dans la paroisse St. Landry, à Opelousas, en Louisiane. Jeanne Grégoire, «Les Acadiens dans une seconde patrie: la Louisiane" Revue d'histoire de l'Amérique française, 16, 2 (septembre 1962): 262 . 
cielles portèrent même sur la possibilité de conquérir la Nouvelle Espagne dans son entier ${ }^{40}$. Les autorités espagnoles ne cessèrent jamais de redouter ces incursions françaises, préoccupation qui colora leur réaction à l'arrivée de l'expédition Mallet.

L'accès des Français au Nouveau-Mexique était fonction du contrôle qu'exerçaient Comanches et Apaches sur les voies navigables plausibles, c'est-à-dire le Missouri, l'Arkansas et la Rivière Rouge ${ }^{41}$. Les Espagnols, de même, tentèrent parfois de pénétrer dans des territoires où les Français espéraient voir s'imposer leur influence. C'est ainsi qu'en 1720, don Pedro de Villasur dirigea une expédition de soldats amérindiens et espagnols à la rivière Platte, dans l'actuel Nebraska. À la recherche des Français, Villasur se heurta plutôt à des Amérindiens hostiles qui mirent sa troupe en déroute ${ }^{42}$. La zone frontière entre la Nouvelle-France et le NouveauMexique demeura ainsi sous contrôle autochtone ${ }^{43}$.

Malgré le but strictement commercial de leur expédition, le compte rendu des frères Mallet aux autorités contribua à conforter les Français dans leurs desseins. Les Mallet mettaient en lumière la fragilité de la mainmise espagnole sur le territoire: «Santa Fe [...] est une ville bastie en bois et sans aucune fortification. Il peut y avoir 800 familles espagnoles ou mulastres... Il n'y a que quatre-vingts soldats de garnison, mauvaise troupe et mal armée à Santa Fe. » Les Français avaient établi de fort bons contacts avec les Indiens Lalitan; leur distribution de présents donnait de bons résultats. "[C]ette nation, assuraient les Mallet au lecteur, seroit toute entière à nous, si nous avions quelque establissement dans le pays ${ }^{44}$. ” Outre la possibilité d'une conquête, les perspectives commerciales entre la Louisiane et le Nouveau-Mexique se révélaient attrayantes.

De Louisiane, le gouverneur Bienville et le commissaire-ordinateur Edmé-Gatien Salmon confirmèrent au ministre, en France, le fait que l'exploration Mallet n'avait pas été entreprise sous égide officielle: «ils avoient esté par les terres, sans que personne fust informé de leur dessein. Nous avons esté aussi surpris que satisfaits de cette descouverte, qui peut devenir un objet très important pour la colonie. » Il ressortait clairement,

40. Charmion Clair Shelby, dir., "Projected French Attacks upon the North Eastern Frontier of New Spain, 1719-1721 », Hispanic American Historical Review, 13 (1933): 457-472.

41. H. E. Bolton, op. cit.

42. Oakah L. Jones, Jr., Pueblo Warriors and Spanish Conquest (Norman: University of Oklahoma Press, 1966), 101.

43. Henry Folmer, Franco-Spanish Rivalry in North America, 1524-1763 (Glendale, CA: Arthur H. Clark, 1953), 288.

44. «Extrait du Journal de ce voyage», dans P. Margry, op. cit., 459-460. 
de ce voyage, que les relations commerciales demeuraient envisageables, bien que difficiles. L'attrait des mines d'argent suscita, de nouveau, un grand intérêt: "Ils disent mesme qu'on leur en a fait voir une, à trois quarts de lieue de la ville [de Santa Fe], et que, si les Espagnols avoient le débouché de l'argent, ils en tireroient; ils les mettroient bientost en valeur $^{45}$. " Les Mallet reçurent mission d'effectuer un nouveau voyage au Nouveau-Mexique en 1741, cette fois sous la gouverne de André Fabry de la Bruyère, mais le groupe se heurta à des difficultés et rebroussa che$\min ^{46}$. Pierre Mallet allait y retourner des années plus tard, en 1750, porteur d'une lettre probablement destinée à Moreau ${ }^{47}$.

\section{L'INQUISITION}

Longtemps avant le retour de Pierre Mallet au Nouveau-Mexique, Moreau avait été exécuté pour crimes contre l'État. Les accusations retenues contre lui figurent dans un long document contenant les témoignages de nombreuses personnes qui, dans la région, avaient entendu parler de ses faits et gestes. Le document s'ouvre sur une plainte adressée par don Gaspar Domingo de Mendoza, gouverneur du Nouveau-Mexique, au señor Joseph Carrillo y Bierme, officier de l'Inquisition à Mexico. Le gouverneur y raconte que ses soldats s'apprêtaient à exécuter Louis-Marie lorsque parvint une lettre de frère Pedro del Pino. Ce moine, agent de l'Inquisition mexicaine au Nouveau-Mexique, demandait de surseoir à l'exécution parce que les crimes profanes de l'individu avaient seuls fait l'objet d'une enquête, et non ses crimes de nature religieuse.

Le désir du gouverneur de ne pas retarder l'exécution tenait probablement à sa connaissance de la culture judiciaire de cette époque. Comme l'explique Charles Cutter, les codes juridiques de la colonie n'autorisaient, en matière profane, aucun appel dans les cas de crime grave. En fait, dans le cas rarissime d'une telle occurrence, le tribunal renvoyait la cause à la Audiencia (cour) qui n’avait préséance sur lui qu'avant qu'il rende décision ou sentence. La décision du tribunal était donc finale et rien ne l'obligeait à se conformer à celle du tribunal supérieur. Dans le territoire du

45. «Avantages d'un commerce avec Santa-Fé. Lettre de MM. Bienville et Salmon», ibid., 466-467.

46. Martha Royce Blaine, "French Efforts to Reach Santa Fe: André Fabry de la Bruyère's Voyage up the Canadian River in 1741-1742", Louisiana History, 20 (printemps 1979): 133-157.

47. Dans C. W. Hackett, dir., Pichardo's Treatise..., op. cit., le témoignage de Pierre Mallet (Pedro Malec) évoque une «lettre adressée à Moreno, un citoyen de la dite ville [de Santa Fe]», 335. La lettre portait-elle sur l'héritage reçu de la mère de Moreau? 
Nouveau-Mexique, l'appel en révision eût été adressée à la Audiencia de Guadalajara $^{48}$.

Mais frère Joseph de Yrigoyen, notaire de l'Inquisition à la Mission de Xemes, ordonna de surseoir à l'exécution pour les raisons invoquées par del Pino. Le cas, disait-il, relevait aussi du Saint Office. Aucun tribunal de l'Inquisition n'exista jamais au Nouveau-Mexique ${ }^{49}$. Le Saint Office, à Mexico, nommait habituellement le supérieur des Franciscains commissaire agissant en son nom dans la province ${ }^{50}$. En 1731, le frère Yrigoyen fut nommé notaire pour l'Inquisition à Santa $\mathrm{Fe}^{51}$. Les Franciscains ayant fréquemment, au XVII ${ }^{\mathrm{e}}$ siècle, fait planer sur les gouverneurs royaux du Nouveau-Mexique la menace d'une excommunication ${ }^{52}$, les relations entre l'Église et l'État n'y avaient pas toujours été de tout repos. Les commissaires de l'Inquisition avaient aussi le pouvoir, dans cette région, de détenir et de poursuivre les intrus français qui pénétraient aux marges de la colonie et causaient des troubles chez les Autochtones ${ }^{53}$. L'intervention de frère Yrigoyen, cependant, n'entre pas dans le cadre de telles pratiques. En fait, il affirma plus tard être intervenu sur la foi de renseignements selon lesquels les ennuis de Moreau avaient pour origine les mensonges d'une femme ${ }^{54}$. Il demeure possible de conclure, de cette remarque, que Moreau fut la victime d'une campagne de ragots dirigée contre un étranger qui avait conclu une alliance peu recommandable avec la fille bigame d'une prostituée. Malheureusement, Yrigoyen ne dit rien de telles alliances. En même temps, il se peut qu'une telle sentence de mort l'ait choqué. La peine capitale était, en effet, chose rare au Nouveau-Mexique et n'était appliquée que pour des crimes d'une extrême brutalité55.

Bien que le document sur lequel s'articule notre article ait été produit par des agents de l'Inquisition, il n'est ni typique ni représentatif des textes qu'on trouve habituellement dans de telles archives. En tant qu' institution, l'Inquisition avait mis au point des instructions précises découlant du

48. Charles R. Cutter, The Legal Culture of Northern New Spain, 1700-1810 (Albuquerque, University of New Mexico, 1995), 53.

49. Marc Simmons, Witchcraft in the Southwest; Spanish and Indian Supernaturalism on the Rio Grande (Flagstaff : Northland Press, 1974), 19.

50. Richard E. Greenleaf, "The Inquisition in Eighteenth-Century New Mexico », New Mexico Historical Review, 60, 1 (1985): 29.

51. AGN, Inquisición, vol. 832, exp. 264/265, 1731.

52. D. Weber, op. cit., 131 ; R. Greenleaf, "The Inquisition... », loc. cit., 29.

53. R. Greenleaf, loc. cit., 41.

54. AGN, Criminal, vol. 577, exp. 10, folio $375 \mathrm{v}$.

55. R. Cutter, op. cit., 138. 
Droit canon ${ }^{56}$ et, bien que Solange Alberro ait montré que plusieurs inquisiteurs aient négligé de s'y conformer étroitement et fidèlement ${ }^{57}$, la procédure prenait habituellement une forme stéréotypée. L'Inquisition dépendait des dénonciations et ne se mettait pas, normalement, en quête de pécheurs à sanctionner; les comisarios (commissaires ou représentants) se contentaient de lire, à intervalles réguliers, les décrets de l'institution et attendaient de la conscience des chrétiens qu'ils se dénoncent ou qu'ils en accusent d'autres d'avoir enfreint la loi de l’Église ${ }^{58}$. Le fait que le frère Pedro del Pino soit ainsi intervenu dans un procès laïque afin d'invoquer la possible dimension religieuse des accusations constitue, par conséquent, un écart à la procédure normale, car aucune dénonciation des activités de Moreau n'était apparemment parvenue aux représentants du Saint Office à Mexico.

Dans le cadre d'une procédure normale, les familiares ou comisarios de l'Inquisition au Nouveau-Mexique auraient reçu au moins trois dénonciations précises et fiables, provenant de personnes non motivées par la haine ou la vengeance ${ }^{59}$. Il fallait de plus, en théorie, que ces dénonciateurs soient de vieille souche chrétienne - c'est-à-dire que leurs antécédents familiaux ne soient teintés d'aucun sang juif ou mauresque, et qu'euxmêmes ne soient pas de conversion récente au catholicisme ${ }^{60}$. Étant autochtones, de nombreux témoins à charge ne satisfaisaient certes pas à cette exigence, outre que les indigènes n'étaient habituellement pas considérés comme des dénonciateurs fiables ${ }^{61}$.

En fait, les dénonciations faisaient partie d'un tissu d'accusations qui prenait forme lorsque plusieurs personnes attiraient l'attention sur un individu de conduite douteuse; le Saint Office examinait alors le dossier et, si les renseignements lui paraissaient suffisamment fiables et assez nombreux, portait des accusations et incarcérait le suspect dans une de ses prisons. Il arrivait souvent que le prévenu porte à son tour des contreaccusations dans l'espoir d'atténuer sa culpabilité et de bénéficier ainsi

56. Richard E. Greenleaf, Inquisición y Sociedad en el México colonial (Madrid: Ediciones José Porrua Turana, 1985), 6.

57. Solange Alberro, Inquisición y Sociedad en México, 1571-1700 (Mexico: Fondo de Cultura Económica, 1988), 35-41.

58. R. Greenleaf, Inquisición..., op. cit., 36.

59 Ibid., 37.

60. Lewis Tambs, "The Inquisition in Eighteenth-Century Mexico», The Americas, 22, 2 (octobre 1965): 168.

61. S. Alberro, op. cit., 26. 
d'une peine plus légère ${ }^{62}$. Il est clair que la cause Moreau ne fut pas conduite selon cette procédure administrative. Les témoins à charge n'étaient manifestement pas impartiaux, pas plus qu'ils n'étaient des chrétiens de vieille souche, et l'on ne peut guère savoir s'ils étaient venus de leur plein gré pour dénoncer Moreau ou s'ils y avaient été invités par les autorités locales. Il se peut que cet écart à la normalité n’ait été dû qu'aux circonstances ou au simple fait que le Nouveau-Mexique - géographiquement très éloigné du gouvernement colonial central - fonctionnait juridiquement de manière différente. Les agents du Saint Office en poste au Nouveau-Mexique, en effet, ne pouvaient facilement consulter leurs supérieurs ou leurs homologues de $\mathrm{Mexico}^{63}$.

Que Moreau n'ait pas été interrogé constitue, à cet égard, un des écarts procéduraux les plus marqués. Suivant la procédure typique, les agents de l'Inquisition arrêtaient le présumé coupable, lequel était invité, en deçà de huit jours passés dans les geôles du Saint Office, à scruter sa conscience pour y découvrir les raisons de son arrestation. Plus tard, les officiers lui donnaient lecture des accusations sans révéler les noms des dénonciateurs $^{64}$. À notre grand dam, cette étape fut omise dans le cas de Moreau, de sorte qu'il nous a été impossible de juger de ses réactions, pas plus que de connaître sa version des événements et les termes dans lesquels furent formulées les accusations. En outre, le sort qu’on lui réserva sortit nettement de l'ordinaire.

En dépit de l'aura de puissance qui entourait l'Inquisition, rares furent les Mexicains qui eurent à composer avec elle; sur trois siècles de régime colonial, à peine $5 \mathrm{p}$. cent de la population fit l'objet d'arrestations; de ce nombre, 2 p. cent furent condamnés et seulement 1 p. cent exécutés ${ }^{65}$. L'exécution de Moreau, cela va sans dire, fut menée selon une procédure laïque et certainement pas selon les prescriptions du Droit canon, auquel cas il aurait été condamné au bûcher.

Le cas Moreau comporte cependant un volet typique : le rapport étroit entre les définitions d'hérésie et de trahison. Comme l'affirme Richard E. Greenleaf, doyen des spécialistes de l'Inquisition, les juristes mexicains parvenaient difficilement à les différencier l'un de l'autre, les deux crimes répondant à des définitions floues et imprécises ${ }^{66}$. Le contexte du XVIII ${ }^{e}$

62. R. Greenleaf, Inquisición..., op. cit., p. 37; S. Alberro, op. cit., 61.

63. S. Alberro, op. cit., 23-24.

64. R. Greenleaf, Inquisición..., op. cit., 37.

65. Ibid., 7.

66. Ibid., 16. 
siècle et les défis posés à l'État par la philosophie antiroyaliste pourraient peut-être sous-tendre les raisons de l'arrestation et de l'exécution de Moreau, mais ces tendances se firent davantage sentir, dans les colonies, vers la fin du siècle. On a soutenu que l'Inquisition, au XVIII ${ }^{e}$ siècle, a accordé une attention croissante aux dossiers politiques au détriment des affaires religieuses, réagissant ainsi à la contestation du statu quo et à la montée du nationalisme mexicain ${ }^{67}$, mais Greenleaf ne voit rien là de nouveau; les hérétiques étaient des traîtres et les traîtres étaient des hérétiques, et il en avait été ainsi depuis les débuts du Saint Office ${ }^{68}$.

Le matériel d'archives ne décrit pas seulement la lutte menée pour établir quelle institution avait compétence dans le dossier Moreau, mais il fournit aussi divers témoignages concernant les actes séditieux et hérétiques du prévenu. En fin de compte, Moreau fut exécuté pour avoir incité à la révolte la population autochtone, c'est-à-dire pour un crime de lèsemajesté et non de lèse-majesté divine, mais la description de ses activités révèle un fascinant alliage de cultures et de formes de religiosité populaire. De tous les témoins à charge, un seul affirma avoir entendu Moreau; tous les autres ne firent état que de ouï-dire. Les paroles de Moreau ne sont jamais rapportées verbatim; en fait, et bien qu'il ait nié certaines des allégations, il lui fut refusé de prendre la parole avant son exécution ${ }^{69}$.

Ses actes et ses propos nous parviennent néanmoins sous forme fragmentaire et nous permettent un aperçu sur sa perception des habitudes locales et sur la réaction des Néo-Mexicains. Parce qu'il était ostensiblement catholique, son comportement hérétique ne tenait ni du paganisme ni du protestantisme, mais d'une adhésion à une forme différente de catholicisme. De ses propos concernant le clergé et les pratiques religieuses, plusieurs s'articulaient sur l'idée selon laquelle la population de Nouvelle-France pratiquait un catholicisme différent mais meilleur. Lors de leur étude de cette présumée hérésie, les agents de l'Inquisition recueillirent les témoignages de dix hommes et d'une femme, au nombre desquels deux alcaldes (juges de paix ou maires), trois Frères, un tambour, un soldat, un Indien et l'épouse d'un charretier. Tous ces témoins affirmèrent avoir entendu dire que Moreau s'était dit plus fort que Dieu. D’autres avaient ouï dire qu'il s'était livré à la magie.

67. L. Tambs, loc. cit., 169.

68. Richard E. Greenleaf, "The Mexican Inquisition and the Enlightenment, 1763-1805 », New Mexico Historical Review, 41, 3 (été 1966): 181.

69. AGN, Criminal, vol. 577, exp. 10, folio 376. 
Il est possible de répartir en quatre catégories les accusations portées contre Moreau: critique des pratiques religieuses, hérésie, pratique de la magie et sédition. Les trois premières constituent des crimes contre l'Église, bien que soit très variable le danger qu'ils aient représenté. Les deux catégories essentielles - hérésie et rébellion - étaient cependant indissociables dans l'esprit de certains accusateurs. Le frère Yrigoyen, par exemple, affirma qu'en condamnant les pratiques religieuses et en tenant des propos hérétiques, Moreau désobéissait à l’Église. Dans le contexte d'instabilité du Nouveau-Mexique, où l'influence de l'Église demeurait limitée dans certains territoires, il invitait les nouveaux chrétiens à renier leur foi. Moreau tentait, indirectement, de détruire l'Église ${ }^{70}$. Dans l'esprit des autorités coloniales, Foi et État étaient étroitement liés, de sorte qu'il n'est pas étonnant que les propos de Moreau aient soulevé des inquiétudes.

En fait, le Saint Office jugea que les crimes de nature religieuse imputés à Moreau ne justifiaient pas un examen plus poussé. Ils n'étaient pas d'une gravité qui puisse provoquer une telle inquiétude. Cette position des agents de l'Inquisition en poste à Mexico traduisait un changement d'orientation qui se précisa durant le XVIII ${ }^{\mathrm{e}}$ siècle. Prenant leurs distances vis-à-vis des simples cas d'emploi de la magie, ces agents concentraient désormais leurs efforts sur ceux qui menaçaient sérieusement l'ordre colonial. Ainsi, bien que les témoignages contre Moreau aient évoqué le Diable à diverses reprises - «la Cruz, solo el Diablo la cargaba para engañar la gente» (seul le Diable a employé la Croix pour tromper les gens) - l'Inquisition pouvait choisir de ne pas discuter plus avant de cette hérésie. Pour les autorités, a pu conclure un historien à propos d'une révolte survenue antérieurement en Nouvelle-Espagne: «Le satanisme était minimisé ou ignoré, non parce qu'il était trop naïf mais, au contraire, parce qu'il était susceptible de mener à l'incrédulité ${ }^{71}$.»

L'intérêt à faire appel à l'Inquisition traduisait peut-être, dans le cas Moreau, l'écart existant, quant aux normes de l'époque, entre les régions périphériques et le centre du pays. D’un autre côté, il se peut que le frère Yrigoyen ait vraiment cru que les accusations contre Moreau avaient pour cause la jalousie ou tout autre sentiment, et qu'il ait ainsi tenté de retarder

70. Ibid., folio 375 .

71. F. Cervantes, "The Devils of Querétaro: Scepticism and Credulity in late seventeenthcentury Mexico », Past \& Present, 130 (1992): 68. Sur la notion de Diable dans la Nouvelle-France du dix-septième siècle, voir Peter A. Goddard, «The Devil in New France: Jesuit Demonology, 1611-50», Canadian Historical Review, 78, 1 (mars 1997): 40-62. 
l'exécution afin d'en avoir le cœur net. Au cours de l'enquête qui suivit, la femme non identifiée, jusqu'alors présente à l'arrière-scène, disparut du décor. Pourtant, le fait que Moreau se soit livré à des pratiques magiques et séditieuses apparaît d'autant plus intéressant que les témoins venaient de lieux relativement distants les uns des autres.

\section{HÉRÉSIE, MAGIE ET RÉBELLION}

En dépit de la notoriété de Moreau à travers le Nouveau-Mexique, l'essentiel des preuves à charge dont font état les documents proviennent d'un seul homme du village de Tesuque, nommé Francisco el Coyote, et qui est presque certainement le Francisco Zuazo, el coyote, qui fut plus tard interrogé. Zuazo fit plusieurs déclarations relatives aux critiques qu'aurait formulées Moreau à propos des coutumes religieuses au Nouveau-Mexique; ces déclarations furent reprises par Francisco de la Concepción González, missionnaire à Tesuque, et Phelipe Tafoya, habitant de Santa Fe. Certaines critiques étaient plus précises que d'autres. Premièrement, il niait la virginité de Marie après la naissance du Christ. Bien qu'il se soit agi là d'un dogme officiel en Nouvelle-France ${ }^{72}$, de même qu'au Mexique, cette virginité en déconcertait plusieurs et Moreau était là en bonne compagnie. Malgré la ferveur des Mexicains envers la Vierge, les arguments spécieux de Moreau n'étaient pas si dangereux.

Deuxièmement, Moreau critiqua la pratique, courante dans le Mexique colonial, de tracer à trois reprises le signe de la croix (sur le front pour l'esprit, sur la bouche et sur le cœur). Pour le frère Cayetano Otero, de la mission de Zuni, cela signifiait que Moreau niait la Trinité. Bien entendu, la Trinité était aussi partie intégrante du dogme en Nouvelle-France. Cependant, la manière de tracer «la marque du chrétien» y était différente, le signe de la croix s'effectuant d'un seul geste, la main droite successivement portée au front, à la poitrine, à l'épaule gauche et à l'épaule droite $^{73}$. Comme les Franciscains comptaient parmi les principaux tenants du dogme de la Trinité, il se peut que leur sensibilité à ce thème les ait portés à exagérer la portée de cette différence. Ils croyaient la Trinité être un "modèle parfait de l'ordre sur terre»; ils y voyaient le modèle du corps politique parfait et de la déférence nécessaire dans une société hiérar-

72. Jean-Baptiste de la Croix de Chevrières de Saint-Vallier, Catéchisme du diocèse du Québec (À Paris: Chez Urbain Coustelier, 1702), 40.

73. Ibid., 483-484. 
chisé ${ }^{74}$. En niant la Trinité ( negaban las tres personas») $)^{75}$, Moreau niait aussi, à leurs yeux, l'autorité de l'Église et celle de l'État. Ce qui n'avait probablement été, pour Moreau, qu'une simple remarque concernant une coutume religieuse différente qu'il estimait surfaite et inutile, devint, aux yeux de frère Cayetano Otero, un propos lourd de sous-entendus hérétiques et séditieux. Les deux parties étaient certes toutes deux de religion catholique, mais leurs pratiques différentes ouvraient un abîme d'incompréhension. En tenant allègrement de tels propos, Moreau entrait, consciemment ou non, en territoire hérétique.

Moreau critiqua aussi d'autres pratiques religieuses en usage dans la colonie espagnole, parfois au seul motif qu'elles différaient de celles de la Nouvelle-France. Selon des témoins, il avait critiqué l'habitude qu'avaient les gens, en Nouvelle-Espagne, de se saluer par un "Ave María». Il qualifia aussi de stupide l'habitude de tracer une croix sur la bouche, expliquant que la bouche ne servait qu'à manger. Il déclara du chapelet qu'il n'était utile qu'à tenir le compte des journées passées en voyage, bien que le chapelet ait été de pratique courante en Nouvelle-France.

Finalement, Moreau se fit extrêmement critique du clergé du NouveauMexique. Bien qu'un témoin ait affirmé que Moreau considérait le frère González comme un $\mathrm{ami}^{76}$, ce dernier déclara avoir entendu dire que le Canadien avait mis en question sa manière de dire la messe ${ }^{77}$. Au pays natal de Moreau, les prêtres disaient mieux la messe. (Ironiquement, le botaniste suédois Pehr Kalm, contemporain de Moreau, exprima une opinion semblable à propos des pratiques religieuses en Nouvelle-France. Pour cet homme de confession protestante, l'emploi du latin par un clergé qui ne le maîtrisait peut-être pas et le récitait devant des paroissiens qui ne le comprenaient pas, privait de sens le rituel religieux. "S'il existe un endroit au monde, où l'on pratique l'office divin comme un opus operatum, c'est bien dans cette région ${ }^{78}$. " La critique de Moreau portait sur les différences dans la manière de célébrer mais ne rejetait pas l'emploi du latin. Reflétant des différences doctrinales, l'érudit suédois eut des mots très durs à l'égard des pratiques religieuses en Nouvelle-France, mais eut

74. Ramón Gutiérrez, When Jesus Came, The Corn Mothers Went Away: Marriage, Sexuality, and Power in New Mexico, 1500-1846 (Stanford: Stanford University Press, 1991), 97-98.

75 AGN, Criminal, vol. 577, exp. 10, fol. 385.

76. Ibid., fol. $385 \mathrm{v}$.

77. Ibid., fol. 386v.

78. Jacques Rousseau et Guy Béthune, trad., Pehr Kalm, Voyage de Pehr Kalm au Canada en 1749 (Montréal: Pierre Tisseyre, 1977), 169, 438 (citation). 
certes la prudence de limiter ses propos à la page écrite plutôt que de les exprimer ouvertement.)

De toutes ces critiques, il semble ressortir que Moreau se refusait à admettre que les pratiques religieuses pussent être à ce point différentes les unes des autres dans deux colonies catholiques. Manifestement, les salutations en forme d'Ave María ne relevaient pas du dogme mais des habitudes locales. Il semble ainsi avoir réagi négativement à des facteurs qui lui rendaient étranger le type de catholicisme du Nouveau-Mexique. Peut-être cet antagonisme tenait-il à sa déception devant une pratique religieuse qui, plutôt que réconfortante parce que familière, lui apparaissait essentiellement étrangère.

Mais les témoignages les plus accablants furent probablement ceux qui lui attribuèrent un propos selon lequel il se disait plus puissant que Dieu. Chacun à sa manière, tous les témoins racontèrent que Moreau ne manifestait aucune crainte des conséquences de ses propos séditieux car, disait-il, il était plus puissant que Dieu. Il avait apparemment déclaré que Dieu était au ciel et lui (Moreau) sur terre, de sorte que lui (Moreau) était le plus puissant. Quelqu'un avait dit au frère Juan Joseph Orinoso, missionnaire à Acoma, que Moreau déclarait «Dios estaba abajo y el arriba ${ }^{79}$ » (Dieu était au-dessous et lui au-dessus). De tels propos eussent été aussi scandaleux en Nouvelle-France qu'ils le furent au Nouveau-Mexique.

Outre ces remarques critiques et hérétiques, Moreau était accusé de se livrer à des activités qu'on ne peut décrire autrement que comme des démonstrations de pouvoir magique. Trois témoins espagnols racontèrent avoir entendu dire que Moreau avait abattu un oiseau en vol en projetant, sans plus, une poignée de poussière dans les airs. Nicolas de Chacey, soldat espagnol de Santa Fe, prétendit que Moreau avait ramassé au sol un bout de bois sec qu'il avait ensuite enroulé comme s'il s'était agi d'une peau ${ }^{80}$. Le frère Juan Joseph Orinoso affirma que le prévenu prit en main une baguette toute sèche, l'enroula autour de son bras et la lança, mais qu'elle demeura sur son corps $^{81}$. Selon ce témoin, en outre, d'autres personnes lui avaient dit que Moreau avait étiré une pierre comme si elle avait eu la consistance de la mélasse. On dit aussi qu'il avait fait voler dans les airs une "gamuza» (sorte de chamois fait d'une peau de chevreuil ou d'ori-

79. AGN, Criminal, vol. 577, exp. 10, fol. 386.

80. Ibid., fol. 384 .

81. Ibid., fol. 386 . 
gnal $^{82}$ ). Il disposait, semble-t-il, quatre bougies sur une gamuza, laquelle acquérait ainsi le pouvoir de s'envoler au loin et même de revenir plusieurs jours plus tard. Sachant que les témoins répétaient des anecdotes racontées par d'autres, il n'est pas étonnant que certains témoignages aient été quelque peu embrouillés. Le frère Cayetano Otero prétendit avoir entendu parler d'épisodes au cours desquels Moreau aurait changé une pierre en gamuza. Manifestement, ce témoin était particulièrement troublé par les maintes choses que Moreau ne craignait pas dire publiquement et les dangers que cela représentait ${ }^{83}$.

Au-delà des truquages d'illusionniste qu'il pratiqua probablement pour impressionner, il est possible que Moreau ait aussi joué, quant à la magie, sur les croyances populaires qui existaient encore au début de l'ère moderne, dont notamment certaines traditions encore vivantes en Nouvelle-France. Bien que la plupart des cas de sorcellerie survenus dans la colonie française se soient réduits à de simples querelles entre particuliers, certaines accusations font état de pouvoirs surnaturels. Charles Raimond dit Passe-campagne, contemporain de Moreau, fut ainsi accusé d'être un sorcier et d'avoir le pouvoir de s'envoler dans les nuages ${ }^{84}$. Il obligea ses détracteurs à retirer, devant notaire, leurs accusations. Dans la version nord-américaine de la légende de la Chasse-galerie, un canot est capable de voler dans les airs après que ses occupants eurent accepté de signer un pacte avec le Diable. L’envolée comme phénomène de magie faisait certainement partie du folklore et des croyances populaires. La magie s'intégrait facilement aux croyances religieuses ${ }^{85}$.

Les excentricités de Moreau en matière de religion n'étaient pas caractéristiques du comportement d'un catholique de Nouvelle-France. Selon les historiens de la religion populaire dans cette colonie, la pratique religieuse y était plutôt traditionnelle, même si certains contestaient parfois l'autorité de l’Église ${ }^{86}$. Des rares cas de sorcellerie mentionnés ici ou là

82. Selon Marc Simmons, les gamuzas étaient "partie intégrante de la vie quotidienne au Nouveau-Mexique ", où on les utilisait comme pièce d'habillement et à diverses autres fonctions ; les habitants des Pueblos étant d'excellents chasseurs, l'approvisionnement en était abondant. Coronado's Land: Essays on Daily Life in Colonial New Mexico (Albuquerque: University of New Mexico Press, 1991), 4-7.

83. AGN, Criminal, vol. 577, exp. 10, fol. 385.

84. Robert-Lionel Séguin, La Sorcellerie au Québec du XVII au XIX siècle (Montréal: Leméac, 1971), 205.

85. C. Podruchny, op. cit., 78-79.

86. Marie-Aimée Cliche, Les pratiques de dévotion en Nouvelle-France. Comportements populaires et encadrement ecclésial dans le gouvernement de Québec (Sainte-Foy: Les Presses de l'Université Laval, 1988). 
dans les archives ecclésiastiques et judiciaires de Nouvelle-France, aucun ne ressemble à celui de Moreau. Pourtant, de telles accusations pouvaient être prises très au sérieux. En 1742, le soldat François-Charles Havard fut accusé, à Montréal, d'avoir commis un sacrilège en utilisant un crucifix comme instrument de divination dans une tentative de découvrir l'identité d'un voleur. Il fut condamné à cinq ans aux galères, sentence ultérieurement réduite à trois ans ${ }^{87}$.

Que les activités présumées de Moreau aient visé à la sédition fut probablement beaucoup plus important que ses dons de magicien. Seul témoin à rapporter ce qu'il avait directement vu et entendu de Moreau, Francisco Zuaso, el Coyote, omit complètement de parler de tours de magie. Son témoignage n'en fut probablement que plus effrayant. Il vaut cependant de noter que Zuaso ne parlait pas très bien l'espagnol, de sorte qu'il est possible que cette difficulté à communiquer ait amplifié les méprises entre les deux hommes. Le 10 juin, Zuaso déclara que Moreau lui avait expliqué comment les gens utilisaient des peaux d'animaux comme moyen de communication afin de déclencher une guerre dans son pays ("en su tierra convidavan para pelear con una gamuza»). Peindre des cercles au centre d'une peau tendue, une pipe faite d'un trognon de maïs et une plume d'aigle teinte en ocre faisaient partie du cérémonial. En tirant à tour de rôle une bouffée de la pipe (chacuaco), les participants acceptaient tous de s'engager dans la guerre ${ }^{88}$. Phelipe Tafoya et Francisco de la Concepción González, qui s'étaient déjà entretenus avec Zuaso mais n'avaient, semble-t-il, eu aucun échange avec Moreau, décrivirent un semblable rituel de guerrier. Interrogé de nouveau le 14 juin, Zuaso répéta ses propos du 10, ajoutant que Moreau avait dit que dans son pays, la sorcellerie ("hechiseria») était excellente et qu'au Nouveau-Mexique tous étaient pauvres. Qui plus est, les Espagnols étaient faits de sang de cochon et de chiens noirs et blancs. Zuaso ajouta croire que Moreau souhaitait tuer le frère González, missionnaire de Tesuque.

Telles étaient les menaces qui inquiétèrent apparemment les autorités. Joseph Yrigoyen, notaire de l'Inquisition, avait antérieurement nommé la personne avec qui Moreau entendait communiquer. Joachim Sánchez, domestique de l'alcalde de Santa Fe, lui avait raconté que Moreau tentait, à San Diego de los Xemes, d'amener les Amérindiens à se révolter. Moreau avait posé au sol une gamuza sur laquelle il disposa quatre bougies. Les

87. Ollivier Hubert, Sur la terre comme au ciel: La gestion des rites par l'Église catholique du Québec (fin XVII - mi-XIXe siècle) (Québec: Les Presses de l’Université Laval, 2000), 70-72.

88. AGN, Criminal, vol. 577, exp. 10, fol. 379. 
chandelles s'élevèrent dans les airs et la gamuza s'envola vers la province de Moqui, territoire sur lequel l'autorité des Espagnols demeurait précaire $^{89}$.

Selon certains ouï-dire, dont le contenu chevauche la frontière alors imprécise entre magie et sédition, Moreau avait appris d'un cheval blanc que son peuple (les Français) étaient en voie d'arriver au NouveauMexique. Il se peut, bien entendu, que Moreau n'ait fait là qu'une simple allusion à l'espoir des frères Mallet d'établir des relations commerciales entre le Nouveau-Mexique et la Louisiane. Mais cette communication magique avec un cheval jouait sur l'anxiété qu'entretenait sans cesse la perspective d'une rébellion ou d'une guerre. La population du NouveauMexique étant déjà au fait des activités des trappeurs et des commerçants français, la prédiction de Moreau, selon laquelle "son" peuple arrivait dans la région, n’apparaissait pas si farfelue, même tenue de la bouche d'un cheval. Selon le frère Juan Joseph Orinoso, missionnaire à Acoma, «Il (Moreau) affirma qu’il éliminerait tous les Espagnols parce qu'ils étaient si peu nombreux ${ }^{90}$.» Francisco Zuaso déclara que Moreau avait prétendu pouvoir facilement maîtriser les Espagnols: «con solo menear un pie, acabaria a todos les Españoles y a todos los Yndios" (il était capable, en agitant seulement le pied, de venir à bout de tous les Espagnols et de tous les Amérindiens) ${ }^{91}$. Leonor Montaño, épouse d'un charretier, raconta que son mari avait rencontré Francisco Coyote qui lui avait dit que Moreau lui avait annoncé que s'achevait la domination espagnole: "no trabajeremos sino que seremos señores absolutos les ayudaria que era Más que Dios» («Nous n’aurons plus à travailler, nous serons plutôt les maîtres absolus. [Moreau] nous aiderait car il était plus puissant que $\left.\mathrm{Dieu}^{92} . »\right)$

Il est clair que les tours de magie et les prédictions de Moreau troublaient grandement ceux et celles qui les rapportèrent aux autorités espagnoles. Bien entendu, sa réputation s'étendit surtout grâce aux ouïdire - de sorte qu'elle dépassa probablement son véritable talent d’illu-

89. Ibid., fol. 362

90. Ibid., fol. 385 .

91. Ibid., fol. 379 .

92. Ibid., fol. $384 \mathrm{v}$. Bien que les laïques catholiques de cette époque aient peu fréquenté la Bible, qu'ils connaissaient mal, il est possible que Moreau ait fait référence au cheval blanc évoqué au Livre des Révélations (Apocalypse, 6,2) de saint Jean: «Et voici qu’apparut à mes yeux un cheval blanc; celui qui le montait tenait un arc; on lui donna une couronne, puis il s'en alla vainqueur, et pour vaincre encore.» [ndt: in La sainte Bible, École biblique de Jérusalem, Éditions du Cerf, Paris 1972, p. 1625.] 
sionniste. Outre le contenu hérétique de ses propos, son apparent pouvoir de magicien était susceptible de troubler les Néo-Mexicains à plusieurs égards. Il était accusé de faire appel directement au Diable, ce qui menaçait implicitement la chrétienté et l'ordre social. Comme il était à la fois étranger et marginal, il se peut que les habitants de la région aient estimé moins dangereux de le dénoncer que d'être soupçonnés, avec les conséquences que l'on sait, d'avoir collaboré, à ses activités.

\section{COMPRENDRE MOREAU: LE CONTEXTE}

Les allégations d'incitation à la rébellion chez les Pueblos ont servi de justification à l'exécution de Moreau. Rien ne prouve que ces présumés appels à l'insurrection aient amené l'une quelconque des populations autochtones du Nouveau-Mexique à prendre les armes ou même à y songer. Pourtant, Autochtones et habitants d'ascendance espagnole estimèrent que ces propos séditieux constituaient un grave danger.

Si les critiques de Moreau à l'endroit de la religion populaire découlaient de ce qu'il avait connu en Nouvelle-France, le rituel guerrier dont il faisait état renvoie plutôt à son séjour à l'intérieur du continent. En évoquant une peau de chevreuil, une pipe et des plumes comme prélude à la guerre, il décrivait un cérémonial étranger aux pratiques françaises. Étranger aussi aux habitudes des Iroquois, qui étaient en rapport si étroit avec la colonie française et chez qui l'échange de ceintures wampum tenait un rôle central dans le déclenchement des hostilités. Dans le mode guerrier des Iroquois du XVII ${ }^{e}$ siècle, il était habituel de tracer sur les arbres du village une suite de dessins racontant l'expédition guerrière ${ }^{93}$. La méthode décrite par Moreau comporte donc des éléments d'une autre provenance: cercles rouges sur une gamuza ensuite décorée de plumes d'aigle et utilisation d'une pipe. Il s'agirait là, semble-t-il, d'un rituel en usage dans la vallée des Illinois, qui fut le point de départ de l'expédition Mallet. Selon une étude sur le mélange des cultures qui se produisit dans cette région, la pipe à long tuyau (calumet) occupait une fonction symbolique de grande importance: «Les pipes étaient le moyen par lequel s'établissaient des

93. Roland Viau, Enfants du néant et mangeurs d'âmes: Guerre, culture et société en Iroquoisie ancienne (Montréal: Boréal, 1997), 85-86; José António Brandão, "Your fyre shall burn no more»: Iroquois Policy toward New France and Its Native Allies to 1701 (Lincoln: University of Nebraska Press, 1997), 33. 
rapports commerciaux, se tenaient des rencontres de paix et se nouaient de bonnes relations sociales ${ }^{94}$.»

Un récit de la campagne de 1739 contre les Chicasas décrit un cérémonial semblable et en explique la signification:

Le $27^{\mathrm{e}}$ on á trouvé à $5 / 4$ de 1 . de nostre fort une canne de Roseau, dans laquelle estoit passé un morceau de drap Anglois, en forme de sac, ramply de tabac, au haut un epys de mahÿ, et dessous un pau d'ours, le tout passoit dans un cercle. C'estoit cinq sauvages Chis qui avoient apportéz nouvellement ces marques enigmatiques qui toutes signifient la paix, suivant l'interpretation de nos sauvages, et des gens du paÿs. Par les Mahÿ, ils entandent qu'ils veulent manger le même pain, le tabac fumer ensemble, et la peau d'ours dans un cercle, coûcher sous le même touït ${ }^{95}$.

Les objets (peaux d'animaux, pipes et nourriture) signifiaient un désir d'amitié et de collaboration. Le rituel de la gamuza était probablement, chez Moreau, une modification de telles pratiques alors qu'il s'efforçait de nouer des liens avec les Pueblos et tentait possiblement de les allier aux Français dont il aurait cru l'arrivée imminente. A-t-il adapté au NouveauMexique une méthode de communication issue d'un milieu géographique et culturel différent, ce qui le mena à un lamentable échec?

Comme nous l'avons déjà noté, la réaction très sévère des autorités aux menaces plutôt bizarres et probablement inefficaces de Moreau tint probablement au contexte politique afférent à la domination espagnole sur les Indiens et au danger que représentaient les incursions françaises et les raids menés par des groupes autochtones. Mais les composantes de magie que comportaient les propos de Moreau ont probablement, de même, soulevé des inquiétudes, car le recours à la magie avait fait partie intégrante, en 1680, du soulèvement victorieux des Pueblos. En 1733, les autorités avaient jugé, à Isleta, un groupe de femmes accusées de sorcellerie $^{96}$. En 1703, après une première tentative de rétablir l'autorité espagnole au sein de la province de Moqui, les habitants poursuivirent leur résistance. Bancroft a montré que cinq juntas de guerra [conseils de guerre] avaient été tenus contre les Moquis ${ }^{97}$. Cette province constituant un îlot de rébellion perpétuelle, toute allusion à un contact avec elle ne pouvait

94. Tanis C. Thorne, The Many Hands of My Relations: French and Indians on the Lower Missouri (Columbia: University of Missouri, 1996), 63.

95. Officier de l'armée de M. De Nouaille, Journal de la guerre du Micissippi contre les Chicachas: en 1739 et finie en 1740, le $1^{\text {er }}$ d'avril (New York: 1859), 44-45.

96. R. Greenleaf, «The Inquisition...», op. cit., 34; D. Weber, The Spanish Frontier..., op. cit., 116.

97. H. Bancroft, op. cit., 226, 233. 
que terroriser les autorités coloniales. En 1740, la province de Sonora, au sud-ouest du Nouveau-Mexique, fut secouée par une révolte chez les Indiens Yaqui. Ce soulèvement dura six mois, durant lesquels les rebelles pillèrent et détruisirent haciendas, mines, ranches et missions. Deux de leurs chefs, qui paradoxalement n’avaient pas préconisé le recours aux armes, furent exécutés pour l'exemple. Leurs têtes coupées furent exhibées dans toutes les rancherías Yaqui ${ }^{98}$.

S'ils avaient des raisons de se préoccuper de maintenir le calme chez les Amérindiens, les Espagnols en avaient tout autant de se méfier des Français. Les dirigeants croyaient que les commerçants français se livraient à une importante contrebande entre le Nouveau-Mexique et la Louisiane ${ }^{99}$. Mais, plus inquiétant encore, ils croyaient que ces incursions et explorations françaises faisaient partie d'une campagne visant à s'emparer de la région du Rio Colorado. Ils s'inquiétaient aussi des échanges commerciaux menés par les Français avec des tribus autochtones, particulièrement après qu'il eut été prouvé que ces Autochtones menaient des escarmouches en utilisant des fusils français ${ }^{100}$. La guerre espagnole contre les Apaches prenait une tout autre dimension car, conséquence des rivalités entre Français et Espagnols, les Français avaient décidé de vendre des fusils aux Comanches et aux Panis. Cette guerre affaiblit la colonie espagnole et obligea ses dirigeants à consacrer leur énergie à fortifier leurs frontières en y détachant troupes de garnison et cavalerie ${ }^{101}$.

Il est difficile de connaître avec précision les activités de Moreau. Certes, rien ne prouve que les Français ait planifié un assaut général sur le territoire, même si les frères Mallet entendaient répéter leur mission commerciale. Mais si des menaces aussi vagues suscitèrent une réaction aussi vive, en dépit de résultats inexistants (v.g. une révolte, réelle ou planifiée), cela tient probablement à ce que Moreau touchait à deux motifs d'inquiétude présents au plus haut point dans le Nouveau-Mexique du XVIII ${ }^{\mathrm{e}}$ siècle. Il parlait de rébellion à une époque où la colonie n’avait été pacifiée que depuis peu et que le souvenir était encore vif de la révolte des Pueblos et de la pacification qui s'en était suivie. Il semble avoir, ou tout au moins le lui a-t-on attribué, mis l'accent sur un des peuples Pueblos encore en

98. Roberto Mario Salmón, Indian Revolts in Northern New Spain: A Synthesis of Resistance (1680-1786) (Lanham: University Press of America, 1991), 73-74.

99. David J. Weber, The Taos Trappers : the Fur Trade in the Far Southwest, 1540-1846 (Norman: University of Oklahoma Press, 1971), 32.

100. H. Bancroft, op. cit., 233, 226.

101. R. Gutiérrez, op. cit., 148. 
révolte à cette époque - les Moquis. En même temps, il incarnait la menace de l'étranger, même s'il avait épousé une femme du lieu et semblait disposé, à certains égards, à s'installer définitivement au NouveauMexique. À une époque où les Français se rapprochaient de la colonie, commerçaient avec les peuples autochtones environnants et établissaient des postes dans la région, toute référence à l'approche des Français ajoutait à la crainte d'une autre révolte des Pueblos. Les propos hérétiques qu'on attribua à Moreau contribuèrent aussi à nourrir ses propositions séditieuses dans la mesure où, considérés comme sérieux par les Pueblos, ils risquaient d'ébranler leur foi et de leur fournir une autre raison de se révolter. Ils mettaient ainsi en péril l'autorité de l'Église, non parce qu'ils étaient intrinsèquement sacrilèges, mais parce qu'ils s'en prenaient à la pratique religieuse quotidienne des nouveaux convertis et des croyants de longue date, les uns et les autres étant la manifestation tangible du pouvoir de l'Église.

Moins de quatre ans après son arrivée au Nouveau-Mexique, Moreau était devenu un personnage dangereux et menaçant aux yeux des autorités espagnoles qui gouvernaient cette province toujours encline à la révolte. À la différence du bizarre Messie Fou de Durango, qui allait sévir au début du XIX siècle, les activités de Moreau traduisaient un programme, dans la mesure où il prévoyait l'arrivée de soldats français venus à la conquête de la région ${ }^{102}$.

Moreau mourut en 1743, fusillé pour trahison et non pour hérésie, sur la place publique de Santa Fe. Après sa mort, les tentatives d'établissement de liens commerciaux stables entre le Nouveau-Mexique et la Louisiane allaient échouer. En 1750, Pierre Mallet retourna à Pecos en compagnie de trois compatriotes. Les quatre Français furent mal reçus. Détenus par les autorités, ils furent transférés à Mexico et de là en Espagne où l'on suppose qu'ils finirent leurs jours ${ }^{103}$. Conséquence, peut-être, du passage de Moreau, les tentatives françaises d'établir des relations avec le territoire du Nouveau-Mexique ne suscitèrent qu'une crainte de troubles que ne pouvaient admettre ni l'État colonial ni l'Église.

Parti à l'aventure depuis Québec, d'où il franchit les territoires sous influence française et parvint au Nouveau-Mexique, Louis-Marie Moreau dit Coulon avait été transformé par son passage dans les territoires non encore colonisés. S’efforçant de communiquer avec les Amérindiens du Nouveau-Mexique, il créa une mauvaise copie des pratiques autochtones

102. E. Van Young, loc. cit., 406.

103. A. P. Nasatir, «Mallet, Pierre Antoine», Dictionnaire biographique du Canada, vol. 3, (Québec, Les Presses de l'Université Laval). 
de la région des Illinois. Innovation fatale, si tant est qu'il tint vraiment les propos dont on l'accusa, il coupla hérésie et rébellion. Les Espagnols avaient grand peine à les distinguer l'une de l'autre.

Moreau incarnait plusieurs dangers : incitation à employer un cérémonial afin de déclarer la guerre, vagues menaces de volonté française de supplanter les Espagnols dans cette partie de l'Amérique du Nord (perspective confirmée par l'utilisation que firent du rapport Mallet les autorités de la Louisiane), et malaise suscité par la critique, par un nouvel arrivant, des pratiques religieuses en cours au Nouveau-Mexique. Par sa seule présence, Moreau représentait un danger dans cette région frontière et les autorités jugèrent nécessaire de le mettre à mort. Produit de la vie en territoire frontalier, Moreau fut lui-même victime de l'insécurité qui y régnait. Comment son compagnon, Jean-Baptiste Alarie, parvint-il à s'en tirer? Cela demeure un mystère. À une époque d'appartenances culturelles fluctuantes, Moreau interpréta quelques aspects de la culture religieuse populaire de Nouvelle-France, mais semble bien, tout compte fait, avoir été irrémédiablement influencé par ce qu'il avait appris sur le chemin du Nouveau-Mexique. Tenant de la supériorité du catholicisme de Nouvelle-France, il intégra néanmoins, dans ses efforts pour communiquer avec différents groupes d'autochtones, certains volets des croyances et traditions autochtones. Son histoire est celle d'un homme qui accepta l'existence de certaines différences chez les nombreux peuples qui habitaient l'Amérique du Nord, mais qui ne comprit pas vraiment le contexte dans lequel il exprima ces différences. Ses activités prirent la mesure des véritables limites de l’Amérique française.

Traduction: Pierre Desrosiers 